\title{
Microdevelopment of Complex Featural and Spatial Integration with Contextual Support
}

\author{
Pamela L. Hirsch ${ }^{1}$ and Elisabeth Hollister Sandberg ${ }^{2}$ \\ ${ }^{1}$ Department of Psychology, Salem State University, 325 Lafayette Street, Salem, MA 01970, USA \\ ${ }^{2}$ Department of Psychology, Vanderbilt University, 528 Wilson Hall, Nashville, TN 37235, USA \\ Correspondence should be addressed to Pamela L. Hirsch; phirsch@salemstate.edu
}

Received 31 July 2015; Revised 17 September 2015; Accepted 21 September 2015

Academic Editor: Olga Capirci

Copyright (C) 2015 P. L. Hirsch and E. H. Sandberg. This is an open access article distributed under the Creative Commons Attribution License, which permits unrestricted use, distribution, and reproduction in any medium, provided the original work is properly cited.

\begin{abstract}
Complex spatial decisions involve the ability to combine featural and spatial information in a scene. In the present work, 4- through 9 -year-old children completed a complex map-scene correspondence task under baseline and supported conditions. Children compared a photographed scene with a correct map and with map-foils that made salient an object feature or spatial property. Map-scene matches were analyzed for the effects of age and featural-spatial information on children's selections. In both conditions children significantly favored maps that highlighted object detail and object perspective rather than color, landmark, and metric elements. Children's correct performance did not differ by age and was suboptimal, but their ability to choose correct maps improved significantly when contextual support was provided. Strategy variability was prominent for all age groups, but at age 9 with support children were more likely to give up their focus on features and transition to the use of spatial strategies. These findings suggest the possibility of a U-shaped curve for children's development of geometric knowledge: geometric coding is predominant early on, diminishes for a time in middle childhood in favor of a preference for features, and then reemerges along with the more advanced abilities to combine featural and spatial information.
\end{abstract}

\section{Introduction}

The capacity to establish correspondence between a map and a scene involves information processing across multiple dimensions that incorporate object features (e.g., color or detail), categorical and fine-grained spatial coding (e.g., left or right of a landmark or metric distances), and array configuration (e.g., whole-scene arrangements). For instance, to establish map-scene correspondence, it is not enough to identify a red barn in both a map and a scene (object feature match), if the red barn is located on the left side of a fence in the map and on the right side of a fence in the scene (spatial category mismatch). Similarly, to establish correspondence, it is not enough to locate a red barn on the right side of a fence in both the map and the scene (spatial category match), if the red barn is represented in the map as located close to the fence and distant from the tree, but in the scene it is located close to the tree and distant from the fence (fine-grained coding mismatch). Further, to establish geometric correspondence, there must be a link between the holistic spatial relations of items in the map and the holistic spatial relations of items in the scene (map-scene match). Whether layout items are pictorially represented or symbolized in iconic line drawings (which often display visual feature mismatches), featuralspatial integration is ultimately necessary to determine correct map-scene correspondence.

Although in the course of ordinary human development it may appear that the ability to combine featural and spatial information transpires smoothly, research has shown that children initially demonstrate knowledge of object features and spatial properties separately [1] and that the two types of information arise from behaviorally and neurally independent systems [2]. Studies with very young children suggest that spatial sensitivity emerges in the earliest years of life [3]. Evidence from recent experiments with nonhuman animals such as newborn chicks argues for an innate predisposition for geometric coding of the environment that depends on a fundamental system that is shared by human and nonhuman 
animals alike [4]. Encoding of geometry involves the ability to process relationships of distance, angle, and direction, which can pertain to the surfaces of a surrounding enclosure or to the global spatial relations between multiple objects. This differs from the ability to process local features of discrete objects that display nonspatial attributes (i.e., color, shape, size, and texture), which can pertain to features that identify a surface (e.g., a colored wall) or to details that identify an object (e.g., a church steeple). Some recent comparative work suggests that chicks spontaneously reorient by computing subtle geometric variations in the terrain of a three-dimensional layout [5]. Developmental research with humans suggests that young children use geometry and do not automatically combine featural and spatial information. For example, studies have shown that 5-month-olds are able to code location of objects hidden in a sandbox, but they are unable to detect differences in objects retrieved from the same locations [6]; that 1-year-olds (as well as nonhuman great apes) choose spatial strategies over featural strategies for understanding object location $[7,8]$; and that 18 - to 24 month-olds rely solely on geometric cues to locate a hidden object in small enclosed spaces [9].

In contrast to the experiments with very young children, several developmental studies indicate that school-aged children, who already have considerable experience in the world and are learning to use multiple sources of information, become more attracted to the nonspatial features of objects than to strategies that depend on the geometry of a space. These studies suggest that on a variety of spatial decisionmaking tasks children may show that they prefer to rely on features rather than locations. For instance, on an objectsearch task, 3-year-old children's early preference for spatial information reverses and children favor feature-based strategies [7]. Further, studies that ask children to disambiguate a hiding place show that 3-year-olds prefer color to location and 4-year-olds prefer size to location [10]; experiments that test 7- to 11-year-olds indicate that object feature information often results in systematic biases in location memory [11], and studies that test 3 - to 12 -year-olds on a picture-environment correspondence task find that featural information is more salient than spatial position [12]. Taken together, these findings imply that features may predominate in spatial decisionmaking during middle childhood.

Yet, in order to make decisions about map-scene correspondence, it is necessary to acquire the ability to combine information about the features of objects and the spatial relations of objects, despite the fact that nonspatial and spatial information often compete for attention. Error patterns may arise due to the separate advancement of information about the featural identities of objects and the configural relations of objects. If children's attention becomes captured predominately by the features of objects, their representations of spatial information may get skewed such that intermediary internal representations give rise to incorrect spatial strategies [13]. Maps may be used to interpret how children are thinking about object features and spatial relations. A pivotal question in the present paper is whether development of children's ability to combine featural and spatial information in order to make correct map-scene matches proceeds along an age-based trajectory or clusters around salient intermediary strategies regardless of age.

The ability to represent spatial information has been traditionally studied in connection with age-dependent developmental capacities. Piaget and Inhelder [14] maintained that spatial development follows a chronologically ordered sequence in which children's spatial decision-making progresses from novice to expert along an age-based, linear pathway. In particular, Piagetian theory proposed that, at approximately 8 or 9 years old, children's abilities undergo qualitative changes with respect to their ability to mentally manipulate spatial information. However, research in developmental variability has suggested that children of all ages use multiple problem-solving strategies in a variety of combinations [15]. This implies that spatial understanding may be present at early ages and spatial miscalculations may appear at older ages. Indeed, research indicates that map skills are active for young children. Children are able to use symbolic functioning at 3 years of age as they form a dual representation of maps and the spaces they stand for [16]. Even young children are able to mentally transform the scenes they encounter. Three-year-olds can interpret aerial pictures as representations of scenes [17, 18], 4-year-olds can understand the relation between a map symbol and its environmental referent $[19,20]$, and children from 4 to 7 years old can form an integrated mental representation for locations of unseen objects [21]. Nevertheless, studies also indicate that older children exhibit many difficulties in mapping a representation to a space. Research shows that 5to 7-year-olds often mistake information cues when vantage point shifts $[22,23], 6$ - to 9-year-olds may misinterpret the intention of map iconicity [24], and 9-year-olds sometimes misjudge configuration in scenes that are occluded at eye level [22].

The present study probed 4- through 9-year-old children's understanding of featural and spatial relations via their choice of the best map to depict a provided scene. The choice of symbolic representations (i.e., maps and photographs) to test strategy shifts in development was based on the theoretical idea that photographs and map representations can provide a "big picture" perspective on spatial relations of the environment that is not as readily available in direct navigational experience [25]. Investigation of how these symbolic representations are used can demonstrate children's spatial cognitive understanding by indicating how they think about spatial relations that have not been experienced directly, that is, how they mentally represent a view of a scene in the absence of navigating that scene (see [5] in regard to unsuccessful effects of matching a real-world view with a two-dimensional snapshot image and see [26, 27] for some qualifications in studies using map-based navigation with $2 \mathrm{D}$ maps of 3D surface layouts). Drawings were not employed to study spatial symbols because they can be confounded by children's limited drawing skills [22]. The central goal of the current work was to investigate the relationship between age and the developmental path of featural-spatial strategy choices, with a focus on the types of intermediate strategy skill components children choose when the task requires complex spatial decision-making. Although variability in children's 
featural and spatial preferences was predicted, it was also expected that age would be a determining factor and that older children would be more likely than younger children to combine featural and spatial components and make correct map-scene decisions. In contrast to studies that measure just age-related successes or failures, this study also explored variability and transitions in spatial strategies across middle childhood. Further, the effect of contextual support on strategy choices was considered by comparing children's trialby-trial performance in baseline and supported conditions and by examining microdevelopment with support.

\section{Method}

2.1. Participants. Forty-eight children were recruited from Boston metropolitan area daycare centers and elementary schools in culturally and socioeconomically diverse neighborhoods. One child was excluded due to procedural error. Forty-seven children (29 boys, 18 girls) were tested: age 4 $\left(n=3, M_{\text {age }}=4.5\right)$, age $5\left(n=7, M_{\text {age }}=5.3\right)$, age $6(n=13$, $\left.M_{\text {age }}=6.3\right)$, age $7\left(n=3, M_{\text {age }}=7.2\right)$, age $8\left(n=14, M_{\text {age }}=\right.$ $8.4)$, and age $9\left(n=7, M_{\text {age }}=9.6\right)$. A written informed consent was obtained from each child's parent/guardian and children provided verbal assent before testing.

2.2. Stimuli. Ten stimulus sets were created. Each set consisted of a photographed scene (see Figure 1) with four map options (see Figure 2). The photographed scenes in each stimulus set included indoor and outdoor settings that were built from such items as dollhouse furniture, model railroads, and toy farm buildings. Scenes were photographed from a viewing angle of approximately $30^{\circ}$ and clearly revealed all items throughout the depth of the scene in such a way that the front and top surfaces of the items were visible. Photographs measuring approximately $9^{\prime \prime}$ by $8^{\prime \prime}$ were matted to construction paper measuring $12^{\prime \prime}$ by $9^{\prime \prime}$. Each map in the stimulus set was a hand-sketched simple, iconic line drawing that depicted the photographed scene from a survey perspective. The iconic line drawings were suggestive of the items shown in the photograph, but these icons bore little visual resemblance to the real objects in the scenes except when the feature being manipulated was made salient in the map. The four maps in a stimulus set, each measuring $4^{\prime \prime}$ by $4^{\prime \prime}$, were matted onto one sheet of construction paper that matched the accompanying photograph's mat in size and color. Each scene photograph and each array of four maps (scale roughly 1:4) were presented in clear plastic document pockets. Maps were unaligned (with either the photograph or with each other) in order to provide a rigorous test of children's spatial relations abilities.

Each stimulus set contained one map that correctly depicted spatial relations of the scene elements and three maps that did not preserve the correct spatial relations of the scene but were designed as foils to systematically make salient one of five featural or spatial components that are known to attract the young child's spatial representational activities $[10,28-30]$. Scene components were delineated by the (relatively) nonspatial strategies of color, detail, and

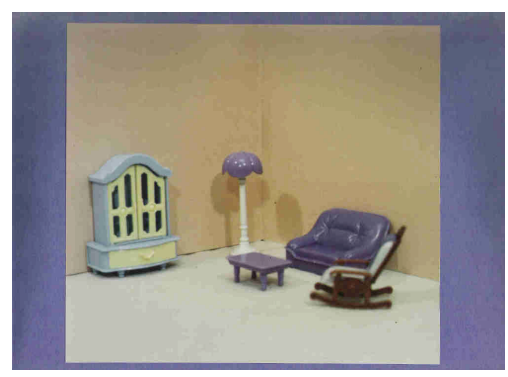

FIGURE 1: Example of a photographed indoor scene used in a stimulus set.

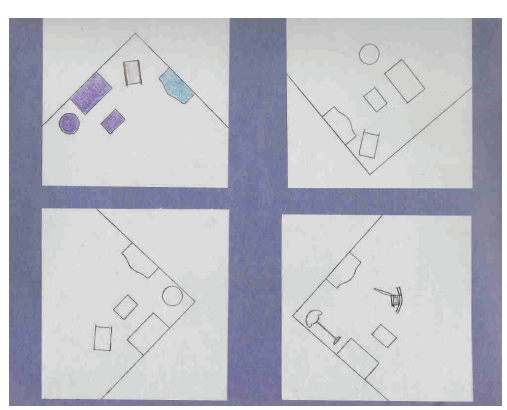

FIgURE 2: Example of the four map options that corresponded to the indoor scene used in the stimulus set example. Beginning top left and moving clockwise, the map choices are Color Foil, Metric Foil, Perspective Foil, and Correct Map.

perspective and by the spatial strategies of landmark, metric coding, and correct spatial relations. A correctly configured map was presented as an option on all ten trials. Each of the map-foil types was seen six times and was paired with all other types an equal number of times. Each map belonged to one of six categories of spatial strategies: (1) Color Foil: elements of the map were colored to match elements in the pictured scene, thereby increasing salience of the color feature despite incorrect location within the spatial layout. (2) Detail Foil: small, distinctive details were included in the depiction of scene elements, thereby increasing salience of physical similarity with particular object features despite incorrect location in the spatial layout. (3) Perspective Foil: an element of the map was drawn from an incorrect perspective that made it transparently identifiable. For example, a tree might be shown from a frontal perspective despite an overhead depiction of the scene. (4) Landmark Foil: a landmark from the scene (such as a prominent building) was drawn in the correct location with respect to the frame of the map, while other distal elements were incorrectly arranged. (5) Metric Foil: objects were drawn in the wrong places but distances were preserved. (6) Correct Map: all elements of the scene were presented in correct spatial relations. 


\subsection{Procedure}

2.3.1. Baseline Condition. Each child was tested individually in the school environment. After greeting the child, the experimenter introduced the task by stating that they would be playing a map matching game. Practice trials were conducted to ensure that the child was familiarized with the materials and instructions in the task. The experimenter asked if the child knew what a map was and closed with the statement "maps are drawings of places that show us where things are in exactly the right places."

A photographed scene was placed on the table and shown to the child. The experimenter instructed the child to look carefully at all of the things in the picture. A map group was then placed next to the photograph and the child was told the following: "one of these maps (pointing to each of four map choices in turn) is the right map for this place (pointing to the photograph). It shows where things are in the right places. Can you show me which map is the right map for this place?" This procedure was repeated for each of the 10 map-scene stimulus sets. Children were permitted to move the maps and the photographed scene. Questions were answered truthfully, but no information was given disclosing spatial information or information about the correctness of a choice. Thus, children arrived at responses that were unmediated. The order of presentation was counterbalanced across participants. The experimenter recorded each child's individual response on each trial as either the color map (c), the detail map (d), the perspective map (p), the landmark map (l), the metric map (m), or the correct map (r).

2.3.2. Support Condition. Participants from the baseline condition went into the support condition in order to explore whether contextual support (i.e., scaffolding, whereby experienced persons (e.g., adults) provide assistance to inexperienced persons (e.g., children) with the aim of helping them to master more complex tasks than they could achieve alone [31]) enabled children to reveal unexpressed higher-order spatial thinking. A second group of ten stimulus sets were created as matched representational pairs to those used in the baseline condition. As in baseline testing, each stimulus set was comprised of one map that correctly depicted spatial configuration and three maps that did not preserve the correct spatial configuration but that made salient one of the five attractive featural or spatial components. A correctly configured map was presented as an option on all ten trials, and each map-foil was seen six times and was paired with all other types an equal number of times.

Children were tested several days after baseline sessions and under the same conditions. The task was reintroduced, and children were told that this time the experimenter would give hints for how to pick the right map. Practice instructions explained the "tricks" that made three maps wrong and the correct correspondences in the fourth map. During test trials the experimenter asked questions that highlighted spatial relations and that elicited a child's self-explanation, which is a known facilitator of cognitive development $[32,33]$. For example, when a child gave a response, the experimenter inquired whether that map showed the items of the photographed scene "in exactly the right places." If the child answered "no," the experimenter then asked the child to explain why and to tell which map "did show things in the right places." The experimenter sometimes provided explanatory feedback, another known facilitator of cognitive change [33]. For instance, the experimenter pointed out when a chosen map showed one building from the side and others from the top. Or, the experimenter indicated if a chosen map showed a table next to a sofa when the scene showed it in front. Children were asked "is that a problem?" At times, they were reminded that they could turn the maps and the photographed scene. Children were permitted to change their responses after consideration of feedback. Individual responses were coded for strategy preferences across the same six possible choices as at baseline.

\section{Results}

3.1. Baseline Results. A one-way ANOVA was conducted on the number of correct map responses, with age in years (4-, 5-, 6-, 7-, 8-, and 9-year-olds) as the independent variable. When results were split across years, no effect of age was found ( $p s$ $>0.05$ ). The power of the test was increased by collapsing the data into two age groups based on the Piagetian claim that children's reasoning undergoes a qualitative shift at approximately 7 or 8 years of age. An independent samples $t$-test was conducted on the younger group of 4- to 7-year-olds $(n=26$, $\left.M_{\text {age }}=6\right)$ and the older group of 8 - to 9-year-olds $(n=21$, $M_{\text {age }}=8.8$ ), but still no significant effect of age was found for correct responses between the younger children $(M=0.09)$ and the older children $(M=0.10), t(45)=0.19$, ns.

Data were then examined to determine children's differential strategy preferences for complex map-scene correspondence when age was excluded as a factor. That is, children's map selections were examined to ascertain how frequently each strategy was chosen, yielding color (16\%), detail (29\%), perspective (28\%), landmark (8\%), metric (10\%), and correct map (9\%). Chi-square tests were used to compare observed frequencies against expected chance frequencies (a raw chance frequency score was calculated at $15 \%$ for each error strategy and at $25 \%$ for the correct strategy). All strategy selections differed significantly from chance expectancy, $\chi^{2}$ $(5, N=943)=90.1, p=0.000$, except the color strategy (ns). Analysis yielded percentage scores that were above chance levels for the nonspatial strategy preferences of color (by 1\%), detail (by 14\%), and perspective (by 13\%) and that were below chance levels for the spatial strategy preferences of landmark (by 7\%), metric (by 5\%), and correct map (by 16\%) (see Figure 3). Notably, these results showed that children's baseline performance strongly favored nonspatial strategies over spatial strategies. Specifically, analysis showed that children's complex map-scene decisions relied significantly on recognizable object details (first) and identifiable object perspectives (second), children significantly avoided landmark or metric elements and correct spatial relations, and children were comparatively neutral about color as a relevant attribute. 


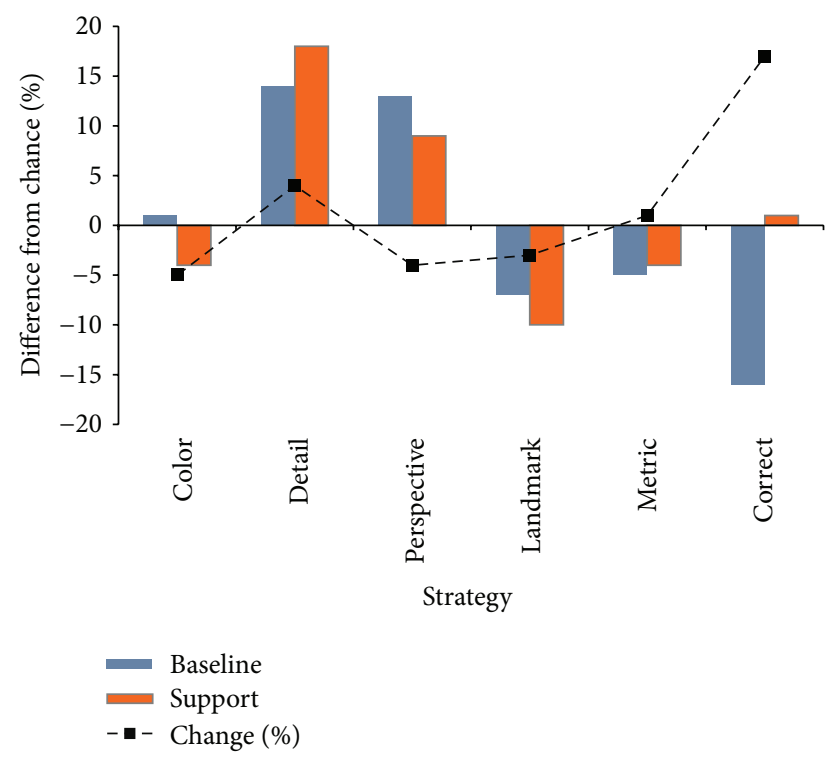

FIGURE 3: Baseline and support percentage scores compared to chance ("0"). The dash line shows percentage score changes from baseline to support.

3.2. Support Results. A $2 \times 6$ (condition $\times$ age) betweensubjects ANOVA was conducted on correct responses and revealed no significant main effects of condition or age in years and no interaction effects ( $p s>0.05$ ). As in the baseline condition, support data were collapsed into a younger group (4 to 7 years old) and an older group ( 8 to 9 years old) based on the idea that spatial reasoning undergoes qualitative changes at approximately 7 to 8 years of age. An independent samples $t$-test was conducted on the younger and older age groups, but no significant effect in correct responses was found between the younger group $(M=0.14)$ and the older group $(M=0.19), t(45)=0.77$, ns. Thus, again, no significant effect of age was found for correct responses. However, a paired samples $t$-test that examined differences in correct responses between baseline $(M=0.09)$ and support $(M=$ 0.16 ) conditions was significant; $t(46)=2.0, p=0.05$. These results suggest that whereas age did not predict accurate mapscene correspondence, correct map responses for the entire group of children regardless of age did improve significantly when contextual support was provided.

Data were then analyzed to determine children's differential strategy preferences when age was excluded as a factor, yielding color (11\%), detail (33\%), perspective (24\%), landmark (5\%), metric (11\%), and correct map (26\%). Chisquare tests were used to compare observed frequencies against expected chance frequencies. All strategy selections differed significantly from chance, $\chi^{2}(5, N=942)=79.1$, $p=0.000$, except for correct map (ns). Analysis yielded percentage scores that were above chance levels for detail (by $18 \%$ ), perspective (by 9\%), and correct map (by 1\%) and that were below chance for color (by 4\%), landmark (by 10\%), and metric (by 4\%) (see Figure 3).

Additional chi-square tests were conducted to compare strategy choices, regardless of age, between baseline and supported conditions. Significant differences were found for correct maps, color, and landmark, $\chi^{2}(5, N=939)=19.7$, $p=0.001$, but not for detail, perspective, and metric (ns). As shown in Figure 3, comparisons of strategy preferences from baseline to support indicated that with support, children's correct map choices increased significantly to a level that was marginally above chance, which is consistent with results of the paired samples $t$-test; they continued to favor salient object details and object perspective; their preferences for color attributes and landmark components significantly decreased; and their preference for metric coding increased slightly. Thus, with support, children still relied extensively on object details and object perspective for complex spatial decisions, yet their ability to use spatial relations significantly improved.

Because results above indicated that the static ability to choose correct map responses did not differ significantly by age, a focus on correct responses was eliminated. Yet, as children's learning is always in flux, questions remained about how much variation there might be in children's use of intermediary featural-spatial components at different ages. In order to further evaluate the variability of spatial trajectories between children, a "featural-spatial strategy shift score" was calculated as a measure of microdevelopmental change in the distribution of strategy usage between baseline and support conditions. Shifted scores were computed as the numerical difference between the number of each strategy choice made in the supported condition and the number of each strategy choice made in the baseline condition across the 10 trials. Then, negative scores were assigned to the category "shifted to features," scores of " 0 " were assigned to the category "unchanged," and positive scores were assigned to the category "shifted to spatial relations." Overall, results showed that $41 \%$ of children's responses shifted toward features, $13 \%$ of children's responses remained unchanged, and $46 \%$ of children's responses shifted toward spatial relations. Chi-square tests conducted on children's featural-spatial shift categories by age in years indicated significant differences in all shift categories for all ages, $\chi^{2}(10, N=47)=$ 18.7, $p<0.05$, except for 4 -year-olds (ns) (see Figure 4). Results showed that 4-year-olds' scores were distributed equally between the categories $(33 \%, 33 \%$, and $33 \%)$; 5year-olds predominantly shifted toward features (56\%) rather than toward spatial relations $(42 \%)$ or unchanged $(0 \%)$; 6year-olds predominantly shifted toward features (69\%) rather than toward spatial relations $(31 \%)$ or unchanged $(0 \%)$; 7 year-olds predominantly remained unchanged (66\%) rather than shifting toward features $(0 \%)$ or toward spatial relations (33\%); 8-year-olds split equally between features (42\%) and spatial relations (42\%), with some still unchanged (14\%); and 9 -year-olds predominantly shifted toward spatial relations (72\%), with the remainder equally split between features (14\%) and unchanged (14\%).

These findings are in agreement with variability research [34], which suggests that the tidy analysis that produced no significant result between age and correct map responses did not capture the untidiness within the data that can help explain developmental unpredictability across middle 


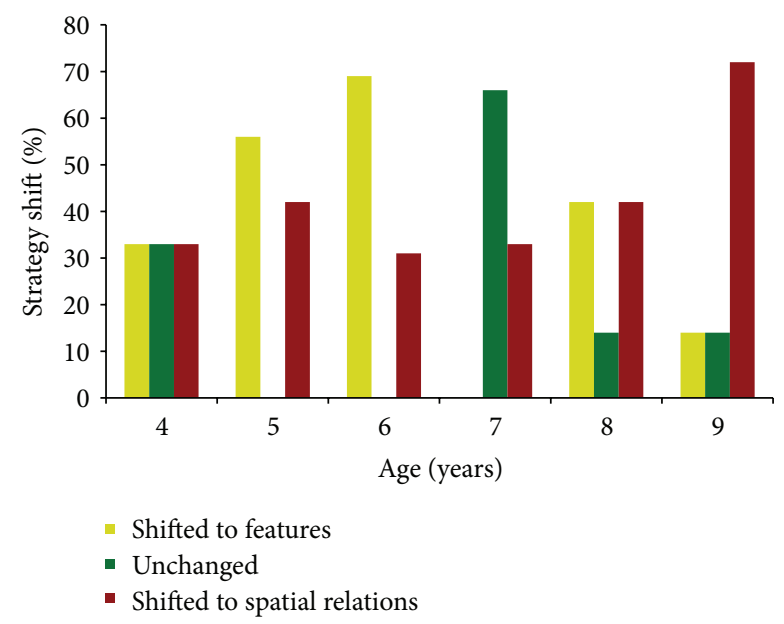

FIGURE 4: Featural-spatial strategy shifts from baseline to support by age in years.

childhood with respect to learning how to combine featural and spatial information.

Microgenetic analysis was used to measure withinchild learning pathways by comparing each child's trialby-trial performance in baseline and support conditions. Data showed that children displayed a dynamic process of variability in completing this complex spatial task. Results indicated that some children who benefited from contextual support showed orderly, stepwise improvement (see Figure 5(a)), but most children's paths toward improvement were characterized by strong fluctuations (see Figures 5(b) and 5(c)). Additionally, results showed that the combination of strategies used was quite distinct for each child. For example, children who benefited equally from support (i.e., they had the same positive shift score) manifested different paths of intermediary strategy movement toward learning about spatial relations (see Figure 5).

Further analysis underscored the notion that featuralspatial reorganization is subject to developmental variability. For instance, children of the same age often showed very different degrees of change (see Figures 6(a) and 6(b) that show shift scores for two 5-year-olds were at the opposite extremes of -6 and +5 ), and children who were at different ages often showed the same degree of change (e.g., see Figures 6(c) and 6(d) that show shift scores for both a 5year-old and an almost 9-year-old were +3 ). Findings also revealed variability for some children who demonstrated spatial relations ability at baseline and regressed during scaffolding (see Figure 6(a)). Similarly, some children showed variability of responses, but their degree of change across 10 trials was unaffected by scaffolding.

Thus, these learning curves for frequency of strategy choices revealed featural-spatial developmental path diversity that was unidentifiable by standard comparisons that used age and correct map responses as variables.

\section{Discussion}

4.1. Baseline and Support Discussions. Based on ingrained traditional models, some improvement in correct map responses across age was anticipated, especially between the youngest and oldest groups; however, contrary to expectations, no reliable age differences were found at baseline. This result was particularly remarkable for older children who often mapped incorrectly and preferred featural information even though they are traditionally considered to be at a later stage of development in which they have established more complex skills. Consistent with newer research, baseline results showed that children's performance across middle childhood significantly favored featural strategies over spatial strategies. Specifically, children relied heavily on object details (first) and object perspectives (second), children systematically avoided landmark or metric elements and correct spatial relations, and children were comparatively neutral about color as a relevant attribute. Baseline findings suggested that children's correct performance on this complex spatial decision-making task was suboptimal and that the ability to combine featural and spatial information that is needed to judge correct maps did not advance significantly across the 4- to 9-year-old age range.

With support, children remained significantly attracted to object details and perspectives, yet their spatial strategy use and correct responses tended to improve. Specifically, although age did not predict correct responses, it was at 9 years of age that children showed a statistically significant transition from the use of featural strategies to spatial strategies. This suggests that many 9-year-olds possessed knowledge potential that they were able to demonstrate when provided with additional scaffolding support. These results are consistent with prior research that found a shift at age 9 in processing fine-grained and categorical combinations [35], as well as with Piagetian theory on qualitative changes in reasoning at approximately 9 years of age. Although results could leave the impression that younger children relied only on features and were not really thinking spatially, investigation of variability from baseline to support showed that cognitive reorganization involved an ebb and flow to the process of learning. For example, during scaffolding, younger children tended to regress to the use of features and older children tended to progress to the use of spatial factors, but strategy changes in each direction signify that learning was taking place [27]. These results support the idea that developmental change emerges as the result of a dynamic system [36], which in this study included prominent instability across middle childhood with regard to the capacity to combine featural and spatial information in a complex scene.

Further, the present microgenetic design provided an opportunity to observe change while it was happening. Trial-by-trial analysis uncovered several microlevel changes that may be understood as transitions in children's spatial thinking and skill acquisition. Investigation of within-child strategy change showed that children who benefited from support learned in distinct ways. For example, children who demonstrated the same degree of change from baseline to support followed a developmental path of fluctuations 

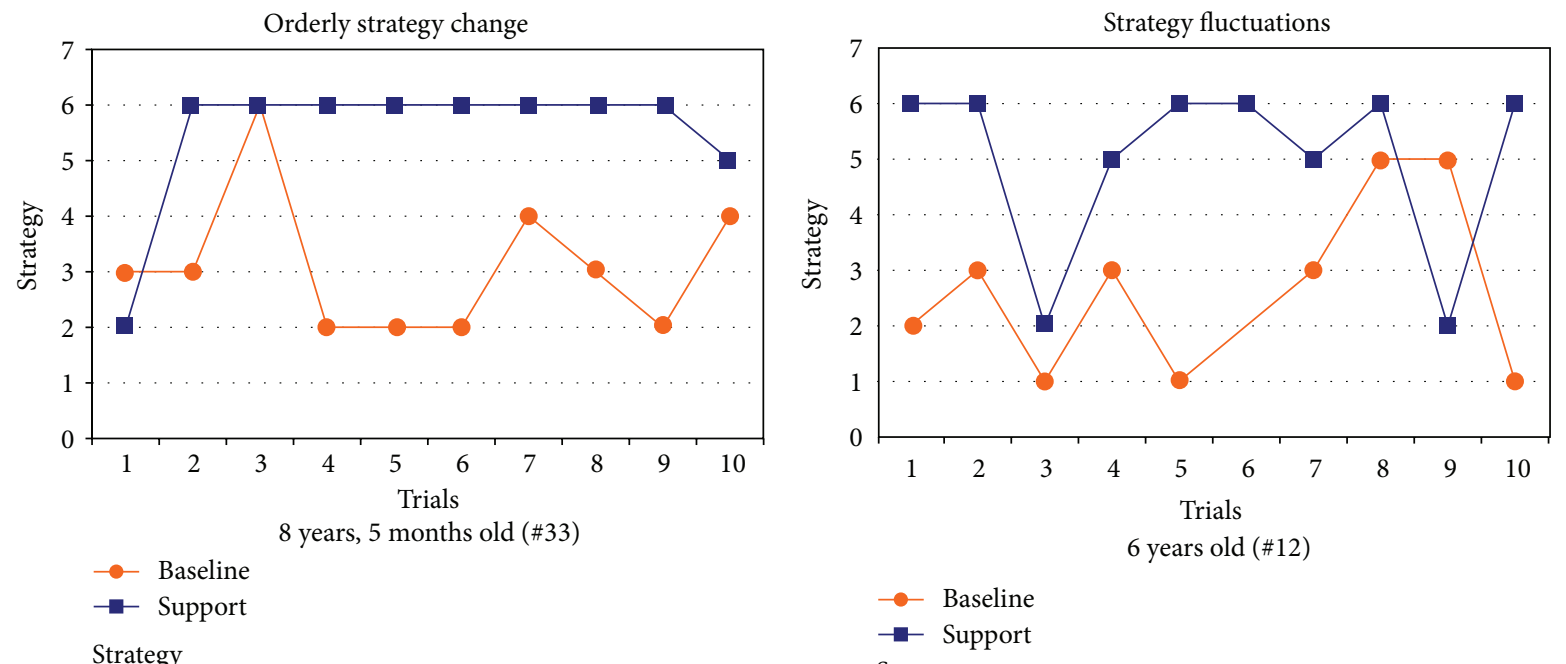

Strategy

(6) Correct

(5) Metric

(4) Landmark

(3) Perspective

(2) Detail

(1) Color

Strategy
(6) Correct
(5) Metric
(4) Landmark
(3) Perspective
(2) Detail
(1) Color

(a)

(b)

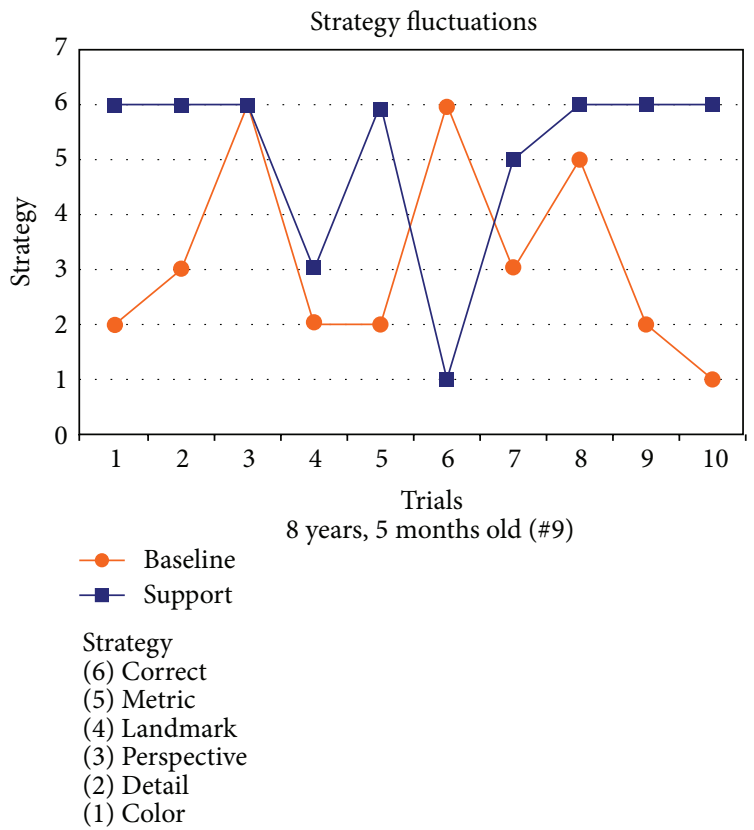

(c)

FIGURE 5: Examples of individual strategy change trajectories from baseline (blue lines) to support (orange lines) in which children benefitted comparably from support. (a) shows a strategy change pathway that was orderly. (b) and (c) show fluctuations in strategy change pathways, but they varied in different ways.

marked by strong individual variability. These results provide evidence against claims that there is a universal sequence to learning. Further comparison of within-child microdevelopment found that children's patterns of strategy change varied independently of age. In particular, findings indicated that degree of change was often dissimilar for children of the same age and similar for children of different ages.
4.2. General Discussion. The present research contributes to the developmental literature by moving beyond emergence of spatial skills in infants and very young children to the study of complex featural-spatial integration during middle childhood. Present results suggest that, between the ages of 4 and 9 years old, complex maps and scenes challenge children's spatial skills. These difficulties might surprise many 

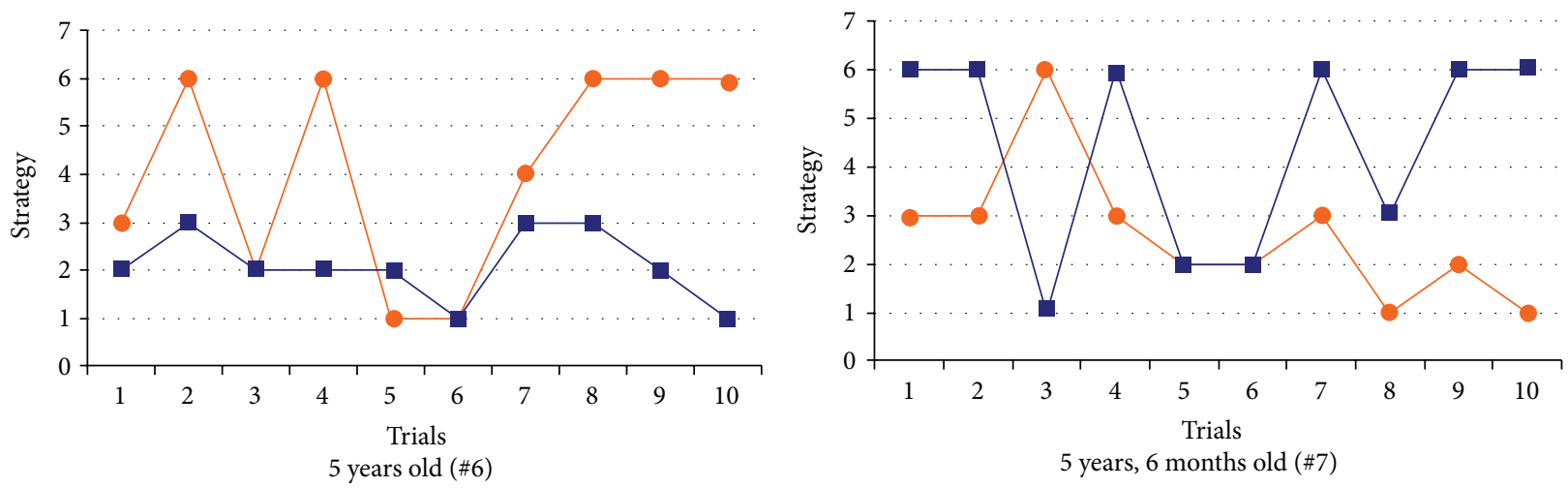

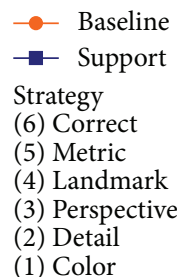

(1) Color

(a)

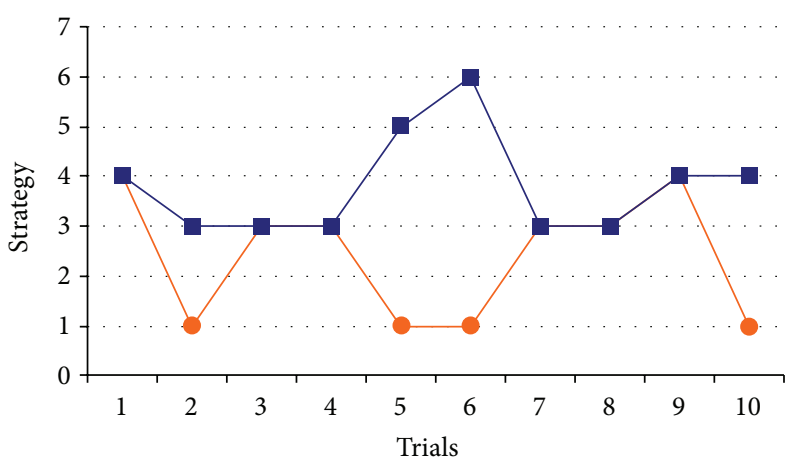

5 years old (\#4)

$$
\begin{aligned}
& - \text { Baseline } \\
& \text {-- Support } \\
& \text { Strategy } \\
& \text { (6) Correct } \\
& \text { (5) Metric } \\
& \text { (4) Landmark } \\
& \text { (3) Perspective } \\
& \text { (2) Detail } \\
& \text { (1) Color }
\end{aligned}
$$

(c)

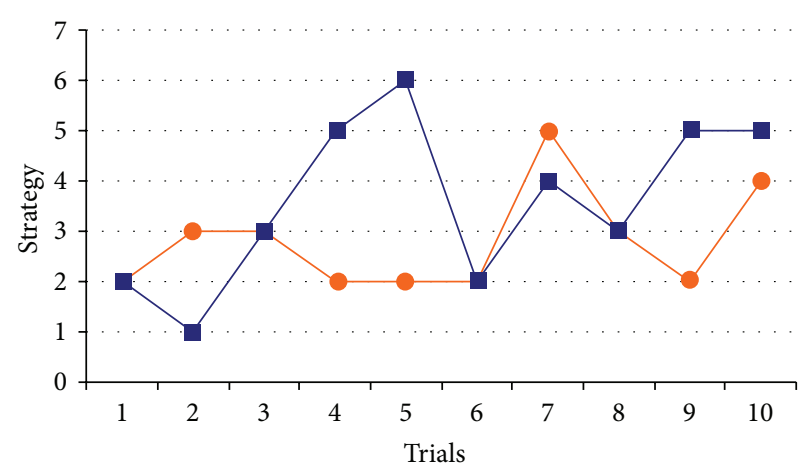

8 years, 10 months old $(\# 40)$ (b)

$$
\begin{aligned}
& - \text { - Baseline } \\
& \text { Strategy } \\
& \text { (6) Correct } \\
& \text { (5) Metric } \\
& \text { (4) Landmark } \\
& \text { (3) Perspective } \\
& \text { (2) Detail } \\
& \text { (1) Color }
\end{aligned}
$$

5 years, 6 months old (\#7)

- Baseline

ategy

5) Metric

3) Perspective

(2) Detail

Figure 6: Examples of individual trajectories of strategy change from baseline to support. (a) and (b) show children of the same age who demonstrated very different degrees of change. (c) and (d) show children of different ages who demonstrated the same degree of change.

parents and teachers who expect children to reason relatively competently about their spatial environment. The current work suggests that one challenge centers on the need to combine feature and spatial compositional elements, which places substantial demands on children's ability to reason about multiple variables that have high levels of relational and symbolic complexity.

The main finding of the present study was that salient object detail and object perspectives were features in scenes that markedly enticed children and that were used significantly more than spatial factors to make choices about map-scene correspondence. This study provides evidence that, across middle childhood, children's featural-spatial strategy choices under complex conditions do not appear to proceed along an age-based trajectory but rather cluster around salient intermediary strategies such as object details and perspectives. Several possible explanations can be proposed for these results. One simple account of featural preferences is that the salient feature captured children's attention and they did not code the entire array. A related explanation is that children did not inspect the line drawings but rather sought the iconicity constraints that preserved a stronger 
resemblance to perceptual features. Children's prominent use of detail-based estimates of location may have relied on cognitive shortcuts that previously proved the details of features reliable on decisions about object individuation, categorization, and disambiguation. Children's prominent use of perspective to estimate location suggests that they are on the cusp of spatial concerns, learning to evaluate different viewpoints and to code projective spatial representations. Consistent with studies of adults [37], it is also possible that children's featural preferences did not signify undeveloped spatial knowledge but instead demonstrated attraction to realistic and elaborate representations over unrealistic and simple ones (line drawings) that are spatially accurate. If children begin life with innate geometric sensitivity and then become attracted to features in middle childhood, they may need to develop the capacity to inhibit their attraction to features as well as the ability to learn more sophisticated ways to reason about space.

Additional findings were based on comparison of performance under baseline and supported conditions, which can help to flesh out the complexities of children's featuralspatial development. The findings clarified that, although age did not predict correct map-scene matches, correct responses for the whole group improved significantly over baseline performance when scaffolding support was provided. Furthermore, analysis revealed that whereas children across the age range relied heavily on featural details and perspective rather than on spatial factors, 9-year-olds' attraction to features began to shift and they were significantly more likely to select maps based on spatial strategies when provided with scaffolding support. Lastly, trial-by-trial analysis indicated that children regardless of age used all the various featural and spatial strategies across testing, demonstrating unique and individual fluctuations in intermediary spatial strategy decisions. In order to update the traditional, linear agebased trajectory and evolve it to a more realistic model of spatial development, it is important to recognize that children are inconsistent and that complex cognitive reorganization involves progression and regression between featural and spatial information. In further research, it would be relevant to refine the complexity and accessibility of stimuli, to gather data on substantial numbers of children, and to compare sex differences in performance.

\section{Conclusions}

The present study suggests that when maps are complex and require the ability to combine multiple sources of featural-spatial information, map accuracy across middle childhood does not proceed along an age-based trajectory but rather clusters around salient nonspatial features such as object details and object perspectives. When children are provided with scaffolding support, correct performance improves significantly. Amidst strong strategy variability overall, microdevelopmental analysis suggests that at 9 years of age with support children can begin to give up their attraction to features and shift toward spatial relations strategies. Taken together with studies showing that young children are predisposed to geometric sensitivity, the present paper suggests that children's development of geometric correspondence between a map and a scene may best be characterized by a U-shaped curve in which geometric coding is predominant early on, diminishes for a time in middle childhood in favor of a preference for object features, and then reemerges along with the more advanced abilities to combine featural and spatial information.

\section{Conflict of Interests}

The authors declare that there is no conflict of interests regarding the publication of this paper.

\section{Acknowledgments}

The authors thank the children who participated in this study and the schools that supported it. They are grateful to the anonymous reviewers for very helpful comments and suggestions.

\section{References}

[1] M. Vasilyeva and S. F. Lourenco, "Spatial development," in The Handbook of Life-Span Development, Volume 1, Cognition, Biology, and Methods, W. F. Overton, Ed., vol. 1, Wiley, Hoboken, NJ, USA, 2010

[2] K. Ferrara and B. Landau, "Geometric and featural systems, separable and combined: evidence from reorientation in people with Williams syndrome," Cognition, vol. 144, pp. 123-133, 2015.

[3] L. Hermer and E. Spelke, "Modularity and development: the case of spatial reorientation," Cognition, vol. 61, no. 3, pp. 195232, 1996.

[4] C. Chiandetti, E. S. Spelke, and G. Vallortigara, "Inexperienced newborn chicks use geometry to spontaneously reorient to an artificial social partner," Developmental Science, 2014.

[5] S. A. Lee, E. S. Spelke, and G. Vallortigara, "Chicks, like children, spontaneously reorient by three-dimensional environmental geometry, not by image matching," Biology Letters, vol. 8, no. 4, pp. 492-494, 2012.

[6] N. S. Newcombe, J. Huttenlocher, and A. E. Learmonth, "Infants' coding of location in continuous space," Infant Behavior and Development, vol. 22, no. 4, pp. 483-510, 1999.

[7] D. B. M. Haun, J. Call, G. Janzen, and S. C. Levinson, "Evolutionary psychology of spatial representations in the hominidae," Current Biology, vol. 16, no. 17, pp. 1736-1740, 2006.

[8] K. Cheng and N. S. Newcombe, "Is there a geometric module for spatial orientation? Squaring theory and evidence," Psychonomic Bulletin and Review, vol. 12, no. 1, pp. 1-23, 2005.

[9] L. Hermer and E. S. Spelke, "A geometric process for spatial reorientation in young children," Nature, vol. 370, no. 6484, pp. $57-59,1994$.

[10] J. M. Plumert and P. Nichols-Whitehead, "Developmental differences in preferences for using color, size, and location information to disambiguate hiding places," Journal of Cognition and Development, vol. 8, no. 4, pp. 427-454, 2007.

[11] A. M. Hund and J. M. Plumert, "Does information about what things are influence children's memory for where things are?" Developmental Psychology, vol. 39, no. 6, pp. 939-948, 2003. 
[12] H. L. Marsh, L. Adams, C. Floyd, and S. E. MacDonald, "Feature versus spatial strategies by orangutans (Pongo abelii) and human children (Homo sapiens) in a cross-dimensional task," Journal of Comparative Psychology, vol. 127, no. 2, pp. 128-141, 2013.

[13] F. L. Dolins and R. W. Mitchell, "Linking spatial cognition and spatial perception," in Spatial Cognition, Spatial Perception: Mapping the Self and Space, F. L. Dolins and R. W. Mitchell, Eds., pp. 1-31, Cambridge University Press, Cambridge, UK, 2010.

[14] J. Piaget and B. Inhelder, The Child's Conception of Space, Norton, New York, NY, USA, 1967-1948.

[15] R. S. Siegler, Emerging Minds: The Process of Change in Children's Thinking, Lawrence Erlbaum Associates, Hillsdale, NJ, USA, 1996.

[16] J. S. DeLoache, "Dual representation and young children's use of scale models," Child Development, vol. 71, no. 2, pp. 329-338, 2000.

[17] C. P. Spencer, N. Harrison, and Z. Darvizeh, "The development of iconic mapping ability in young children," International Journal of Early Childhood, vol. 12, no. 2, pp. 57-64, 1980.

[18] J. M. Blaut and D. Stea, "Studies of geographic learning," Annals of the Association of American Geographers, vol. 61, no. 2, pp. 387-393, 1971.

[19] M. Vasilyeva and E. Bowers, "Children's use of geometric information in mapping tasks," Journal of Experimental Child Psychology, vol. 95, no. 4, pp. 255-277, 2006.

[20] M. Bluestein and L. Acredolo, "Developmental changes in mapreading skills," Child Development, vol. 50, no. 3, pp. 691-697, 1979.

[21] D. H. Uttal and H. M. Wellman, "Young children's representation of spatial information acquired from maps," Developmental Psychology, vol. 25, no. 1, pp. 128-138, 1989.

[22] P. L. Hirsch and E. H. Sandberg, "Development of map construction skills in childhood," Journal of Cognition and Development, vol. 14, no. 3, pp. 397-423, 2013.

[23] L. S. Liben and C. A. Yekel, "Preschoolers' understanding of plan and oblique maps: the role of geometric and representational correspondence," Child Development, vol. 67, no. 6, pp. 27802796, 1996.

[24] L. J. Myers and L. S. Liben, "Graphic symbols as 'the mind on paper': links between children's interpretive theory of mind and symbol understanding," Child Development, vol. 83, no. 1, pp. 186-202, 2012.

[25] D. H. Uttal, "Seeing the big picture: map use and the development of spatial cognition," Developmental Science, vol. 3, no. 3, pp. 247-264, 2000.

[26] Y. Huang and E. S. Spelke, "Core knowledge and the emergence of symbols: the case of maps," Journal of Cognition and Development, vol. 16, no. 1, pp. 81-96, 2015.

[27] A. Shusterman, S. Ah Lee, and E. S. Spelke, "Young children's spontaneous use of geometry in maps," Developmental Science, vol. 11, no. 2, pp. F1-F7, 2008.

[28] D. H. Uttal, "Preschoolers' and adults' scale translation and reconstruction of spatial information acquired from maps," British Journal of Developmental Psychology, vol. 12, no. 3, pp. 259-275, 1994.

[29] E. K. Scholnick, G. G. Fein, and P. F. Campbell, "Changing predictors of map use in wayfinding," Developmental Psychology, vol. 26, no. 2, pp. 188-193, 1990.

[30] M. Blades and C. Spencer, "The use of maps by 4-6-year-old children in a large-scale maze," British Journal of Developmental Psychology, vol. 5, no. 1, pp. 19-24, 1987.
[31] E. K. Foster and A. M. Hund, "The impact of scaffolding and overhearing on young children's use of the spatial terms between and middle," Journal of Child Language, vol. 39, no. 2, pp. 338364, 2012.

[32] A. Cheshire, L. J. Ball, and C. Lewis, "Self-explanation, feedback and the development of analogical reasoning skills: microgenetic evidence for a metacognitive processing account," in Proceedings of the 27th Annual Conference of the Cognitive Science Society (CogSci '05), B. G. Bara, L. W. Barsalou, and M. Bucciarelli, Eds., pp. 435-441, Lawrence Erlbaum Associates, Mahwah, NJ, USA, 2005.

[33] R. S. Siegler, "Microgenetic studies of self explanation," in Microdevelopment: Transition Processes in Development and Learning, N. Granott and J. Parziale, Eds., pp. 31-58, Cambridge University Press, Cambridge, UK, 2002.

[34] E. Flynn and R. Siegler, "Measuring change: current trends and future directions in microgenetic research," Infant and Child Development, vol. 16, no. 1, pp. 135-149, 2007.

[35] E. H. Sandberg, "Cognitive constraints on the development of hierarchical spatial organization skills," Cognitive Development, vol. 14, no. 4, pp. 597-619, 1999.

[36] L. B. Smith and E. Thelen, "Development as a dynamic system," Trends in Cognitive Sciences, vol. 7, no. 8, pp. 343-348, 2003.

[37] M. Hegarty, "Cognition, metacognition, and the design of maps," Current Directions in Psychological Science, vol. 22, no. 1, pp. 3-9, 2013. 

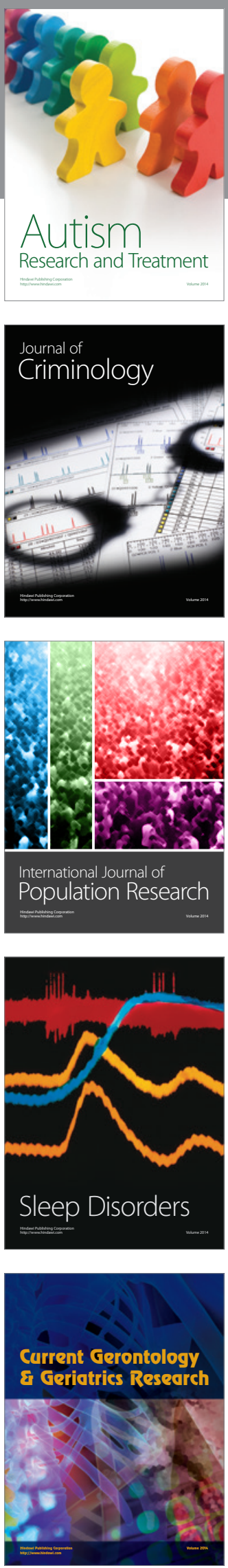
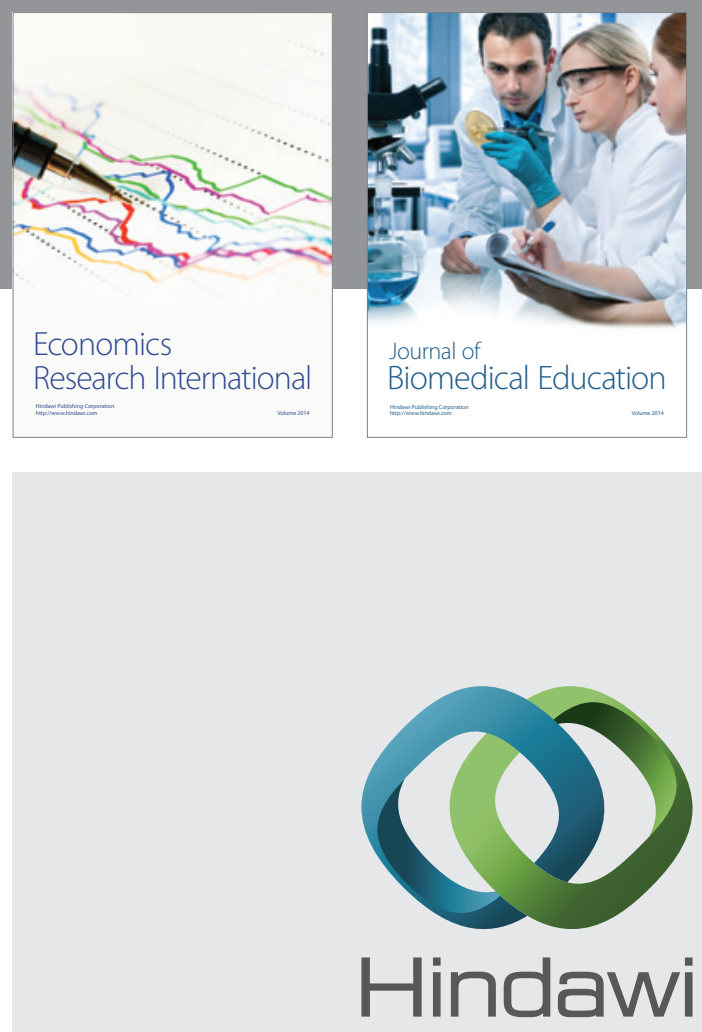

Submit your manuscripts at

http://www.hindawi.com
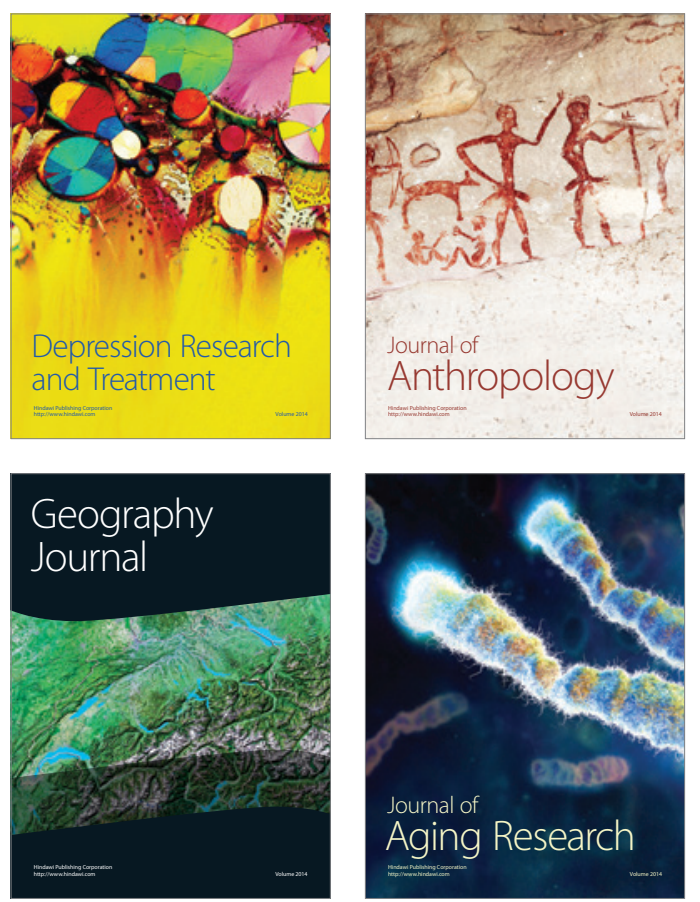
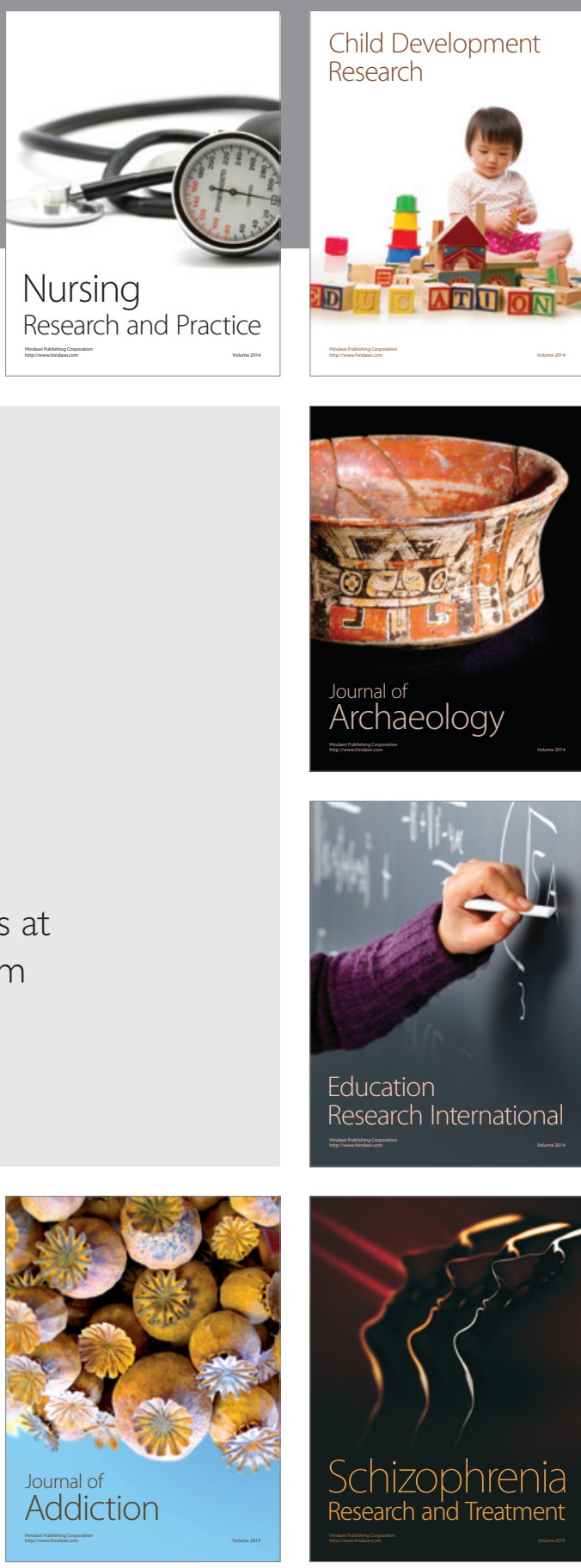

(D)
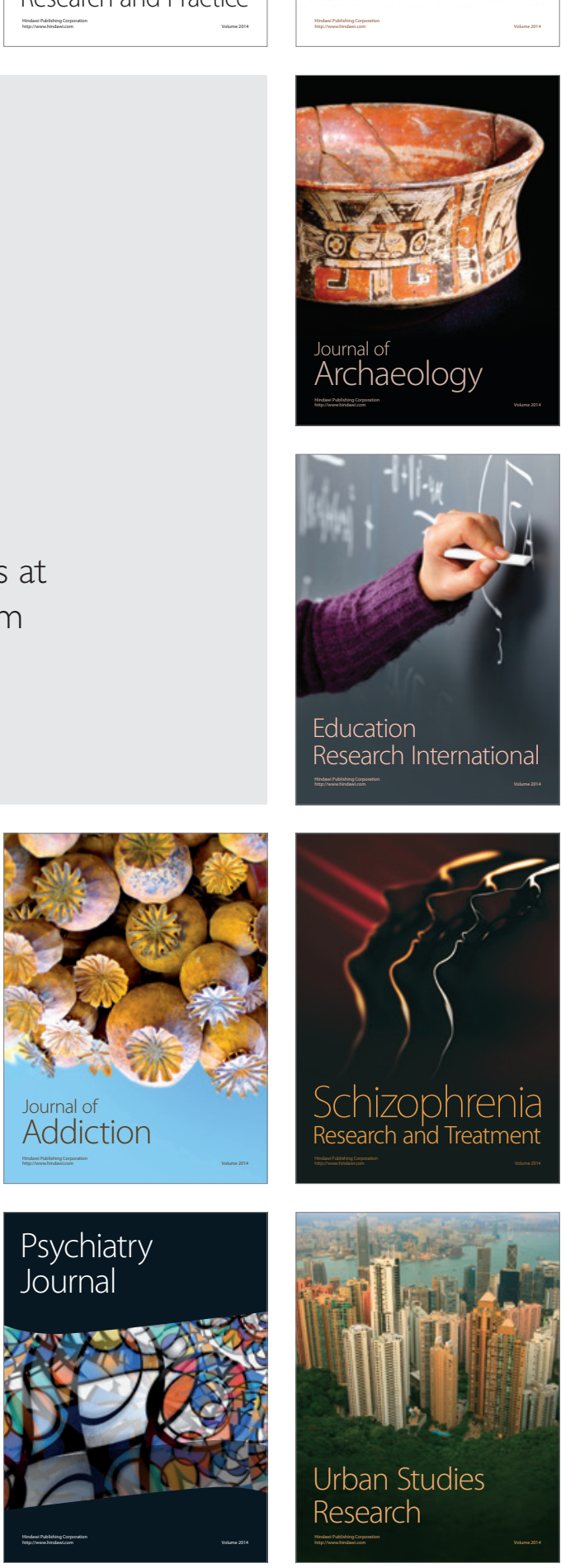